\title{
Proteomic definition of a desmoglein linear determinant common to Pemphigus vulgaris and Pemphigus foliaceous
}

\author{
Alberta Lucchese ${ }^{1}$, Abraham Mittelman², Luciana Tessitore ${ }^{3}$, \\ Rosario Serpico ${ }^{4}$, Animesh A Sinha ${ }^{5}$ and Darja Kanduc*6
}

\begin{abstract}
Address: ${ }^{1}$ Dept. of Odontostomatology, University of Bari, Italy, ${ }^{2}$ Dept. of Medicine, New York Medical College, Valhalla, NY, USA, ${ }^{3}$ DISCAFF, University A. Avogadro, Novara, Italy, ${ }^{4}$ Institute of Clinical Odontostomatology, 2nd University of Naples, Italy, ${ }^{5}$ Division of Dermatology and Cutaneous Sciences, Center for Investigative Dermatology, Michigan State University, East Lansing, MI, USA and ${ }^{6}$ Dept. of Biochemistry and Molecular Biology, University of Bari, Italy

Email: Alberta Lucchese - alucchese@hotmail.com; Abraham Mittelman - ABRAHAM_MITTELMAN@NYMC.EDU; Luciana Tessitore - Luciana.Tessitore@pharm.unipmn.it; Rosario Serpico - rosario.serpico@unina2.it; Animesh A Sinha - asinha@msu.edu; Darja Kanduc* - d.kanduc@biologia.uniba.it

* Corresponding author

Published: 22 August 2006

Journal of Translational Medicine 2006, 4:37 doi:10.1 186/1479-5876-4-37

Received: 28 June 2006

Accepted: 22 August 2006

This article is available from: http://www.translational-medicine.com/content/4/I/37

(C) 2006 Lucchese et al; licensee BioMed Central Ltd.

This is an Open Access article distributed under the terms of the Creative Commons Attribution License (http://creativecommons.org/licenses/by/2.0), which permits unrestricted use, distribution, and reproduction in any medium, provided the original work is properly cited.
\end{abstract}

\begin{abstract}
Background: A number of autoimmune diseases have been clinically and pathologically characterized. In contrast, target antigens have been identified only in a few cases and, in these few cases, the knowledge of the exact epitopic antigenic sequence is still lacking. Thus the major objective of current work in the autoimmunity field is the identification of the epitopic sequences that are related to autoimmune reactions. Our labs propose that autoantigen peptide epitopes able to evoke humoral (auto)immune response are defined by the sequence similarity to the host proteome. The underlying scientific rationale is that antigen peptides acquire immunoreactivity in the context of their proteomic similarity level. Sequences uniquely owned by a protein will have high potential to evoke an immune reaction, whereas motifs with high proteomic redundancy should be immunogenically silenced by the tolerance phenomenon. The relationship between sequence redundancy and peptide immunoreactivity has been successfully validated in a number of experimental models. Here the hypothesis has been applied to pemphigus diseases and the corresponding desmoglein autoantigens.
\end{abstract}

Methods: Desmoglein 3 sequence similarity analysis to the human proteome followed by dot-blot/NMR immunoassays were carried out to identify and validate possible epitopic sequences.

Results: Computational analysis led to identifying a linear immunodominant desmoglein-3 epitope highly reactive with the sera from Pemphigus vulgaris as well as Pemphigus foliaceous. The epitopic peptide corresponded to the amino acid REWVKFAKPCRE sequence, was located in the extreme $\mathrm{N}$-terminal region (residues 49 to 60 ), and had low redundancy to the human proteome. Sequence alignment showed that human desmoglein I and 3 share the REW-KFAK-RE sequence as a common motif with $75 \%$ residue identity.

Conclusion: This study I) validates sequence redundancy to autoproteome as a main factor in shaping desmoglein peptide immunogenicity; 2) offers a molecular mechanicistic basis in analyzing the commonality of autoimmune responses exhibited by the two forms of pemphigus; 3 ) indicates possible peptideimmunotherapeutical approaches for pemphigus diseases. 


\section{Background}

There are over 80 autoimmune diseases known or thought to be autoimmune in nature and, as a group, autoimmune diseases affect approximately $20 \%$ of the population. Multiple mechanisms of autoimmunity induction have been proposed including, among the others, molecular mimicry [1], viral epitope delivery [2], generation of neoantigenic epitopes after posttranslational modification [3], unusual TCR-binding properties that permit autoreactive T cells to escape deletion [4], presence of long regions of extreme structural disorder in the autoantigens [5], cellular injury and release of self antigens, which generate immune responses [1]. However, the mechanisms leading to the breakdown of tolerance against enzyme autoantigen molecules remain poorly understood. The identification and comparative analysis of the autoantigenic immunoreactive determinants could elucidate the molecular basis of the autoimmune disease and offer new immunotherapeutical approaches.

Our labs have proposed that sequence similarity to the host proteome may influence peptide immunogenicity $[6,7]$. According to this hypothesis, only peptide motifs having low similarity to the host proteome have the potential to raise an immune response. Conversely, no immune response can be evoked by antigenic protein fragments that are repeatedly represented in the protein set that form the proteome. In testing this work hypothesis, our current research is focused to the proteomic definition of the epitopic peptide repertoire associated to autoimmune diseases. In practice, based on the assumption that peptide sequences that are scarcely represented in human proteins might provoke autoimmune responses by offering antigenic determinants unknown to (or, better, scarcely encountered by) the immune system, we search for epitopic sequences of human pathogenic autoantigens by, first, selecting for autoantigen fragments not shared with the human proteome and, then, analyzing the immunoreactivity pattern of the not-shared peptides using sera from patients hosting the autoimmune disease. So far, the similarity hypothesis has been validated in a number of different experimental models [6$13]$.

In this study, we used the pemphigus autoimmune skin diseases and the corresponding desmoglein autoantigens as an experimental model [14]. Indeed, pemphigus provides an exemplar paradigm for studying autoimmunity because of its clear-cut antigenic characterization [14,15]. Desmoglein 3 (Dsg3) and desmoglein 1 (Dsg1) are the main autoantigenic target in Pemphigus vulgaris (PV) and Pemphigus foliaceus (PF), respectively $[16,17]$. Moreover, epitope mapping revealed that autoantibodies (AAbs) from PV and PF patients recognize conformational epitopes hosted in the amino terminal ectodomain of
Dsg3 and Dsg1 respectively [16,18]. However, although it has been reported that pemphigus sera contain antibodies against continuous epitopes [16,19], there is a general lack of data on the definition and specificity of Dsg linear epitopes. Therefore, given also that the molecular definition of linear Dsg autoepitopes could contribute to clarify unresolved aspects of pemphigus autoimmunity [20-22], we undertook an experimental study to define the linear epitopic sequences in Dsg3 by using the criterion of nonredundancy to human proteome as a search engine for epitopic peptides.

Here we describe how the search for sequences uniquely present in the human Dsg3 protein led to identifying an amino terminal linear Dsg3 motif that immunoreacts with sera from Pemphigus vulgaris as well as Pemphigus foliaceous.

\section{Materials and methods Computer-assisted analyses}

EC1/EC2 (aa 1-212) portion of human Dsg3 sequence (SWISS-PROT, P32926) was analysed for redundancy to human proteome using PIR protein database (141702 sequences) and peptide match program (pir.georgetown.edu/pirwww) [23]. Sequence alignment was conducted by using SIM - Local similarity program (www.expasy.org) [24].

\section{Sera}

Sera were obtained from the outpatient Dermatology Clinic at the Weill-Cornell Medical College, New York, and Medical College of Wisconsin, Milwaukee. The diagnosis of PV and PF was made on the basis of clinical examination and biopsy as well as indirect IF serum titer. Sera from prostate cancer patients were used as controls.

\section{Peptides}

Peptides were synthesized using standard Fmoc (N-(9-fluorenyl) methoxycarbonyl) solid phase peptide synthesis (PeptidoGenic Research \& Co., Livermore, CA.; Primm srl, Milan, Italy). Peptide purity (>90\%) was controlled by analytical HPLC, and the molecular mass confirmed by fast atomic bombardment mass spectrometry. Peptides used for dotblot immunoassay are: Dsg1 $1_{49}$ ${ }_{60}$ REWIKFAAACRE， Dsg3 ${ }_{36-44}$ EEMTMQQAK, Dsg3 ${ }_{49-}$ ${ }_{60}$ REWVKFAKPCRE, Dsg3 ${ }_{190-204}$ LNSKIAFKIVSQEPA, Dsg3 $373-380$ QVINVREG and Dsg3 ${ }_{518-525}$ NRYTGPYT. The ${ }^{15} \mathrm{~N}$-labelled peptides used for NMR spectroscopy immunoanalysis are: Dsg3 ${ }_{49-60}$ REWVKFAKPCREE, Dsg3 $373-$ ${ }_{380}$ QVINVREG and Dsg3 ${ }_{518-525}$ NRYTGPYT peptides (with ${ }^{15} \mathrm{~N}$-labelled amino acid residues given underlined). Control recombinant proteins corresponding to the EC domains of Dsg1 and Dsg3 [25] were generously provided by Dr. M.S. Lin, Dept. of Dermatology, Medical College of Wisconsin. 


\section{Immuno-blot assays}

Pemphigus serum reactivity against synthetic peptides was tested by immunodotblot assays. Nitrocellulose membrane $(0.2 \mu \mathrm{m}$ pore size, Biorad Laboratories, Milan, Italy) was pretreated with $1 \%$ glutaraldehyde. Dsg3 protein (10 $\mu \mathrm{g})$ or peptides $(4 \mu \mathrm{g})$ were spotted on the activated membranes and immunoassayed with PV or PF AAbs $[8,11,13]$. For Western blot assay, Dsg1 and Dsg3 proteins were resolved on sodium dodecylsulfate-10\% polyacrylamide gel electrophoresis (SDS-10\%PAGE), electroblotted onto PVDF membrane (Biorad Laboratories, Milan), and probed with PV or PF sera.

\section{NMR spectroscopy}

Spectroscopic analyses were carried out on sera pooled and partially purified by precipitation with $40 \%$ saturated $(\mathrm{NH} 4)_{2} \mathrm{SO}_{4}$ (x 2). The precipitate was dissolved in phosphate-buffered saline (PBS), dialyzed against PBS with several changes for $24 \mathrm{~h}$ at $4^{\circ} \mathrm{C}$, then aliquoted and stored at $-20^{\circ} \mathrm{C}$ until assay. NMR spectra of the reaction of the synthetic ${ }^{15 N}$-labelled Dsg3 ${ }_{49-60}$ REW $\underline{\text { KFAKPCRE, }}$ Dsg3 $373-380$ Q $\underline{\text { INVREG or Dsg3 }}{ }_{518-525}$ N $\underline{R Y T G P Y T}$ peptide (with ${ }^{15} \mathrm{~N}$-labelled amino acid residues given underlined) with partially purified AAbs from pooled sera of PV or PF or prostate cancer patients as control were recorded at $298^{\circ} \mathrm{K}$ on a Bruker Avance DRX500WB spectrometer. The spectra were acquired by heteronuclear single quantum correlation (HSQC) experiments as already detailed $[8,11,26]$. We used chemical shift statistics from the full BioMagResBank database, where the calculated statistics are derived from a total of 559392 chemical shifts (www.bmrb.wisc.edu). Sequence-specific correction factor tabulations were applied to backbone ${ }^{1} \mathrm{H}$ and ${ }^{15} \mathrm{~N}$ resonances [27]. Two-dimensional correlated spectroscopy spectra of peptide-AAbs complex were obtained using peptide:AAb ratio equal to $0.1: 30, \mathrm{mg} / \mathrm{mg}$. That is, NMR samples contained either $0.1 \mathrm{mg}$ free $\mathrm{Dsg}_{49}$ ${ }_{60}$ REW ${ }_{525}$ NRYTGPYT) peptide; or $30 \mathrm{mg}$ PV AAbs complexed with $0.1 \mathrm{mg}$ Dsg3 ${ }_{49-60}$ REW $\underline{\text { KFAKPCRE (or Dsg3 }}{ }_{373-}$ ${ }_{380}$ Q $\underline{\text { INVREG }}$ or Dsg3 $318-525$ NRYTGPYT) peptide; or 30 mg PF AAbs complexed with 0.1 mg Dsg3 ${ }_{49-}$ ${ }_{60} \underline{R E W} \underline{V K F A K P C R E}$ (or Dsg3 $373-380$ QIN $\underline{\text { VREG }}$ or Dsg3 ${ }_{518-525}$ NRYTGPYT) peptide; or $30 \mathrm{mg}$ control AAbs complexed with $0.1 \mathrm{mg}$ Dsg3 ${ }_{49-60}$ REWVKFAKPCRE (or Dsg3 ${ }_{373-380}$ Q $\underline{\text { INVREG }}$ or Dsg3 ${ }_{518-525}$ NRYTGPYT) peptide, in $0.5 \mathrm{ml}$ aqueous solution $\mathrm{H}_{2} \mathrm{O} / \mathrm{D}_{2} \mathrm{O}(9: 1, \mathrm{v} / \mathrm{v})$.

\section{Results}

Searching ECI/EC2 Dsg 3 for sequences non-redundant to the human proteome

Since the Dsg antigenic portions recognized by AAbs from PV patients mainly map to the $\mathrm{NH}^{2}$-terminal adhesive domain9 $[18,28]$, this preliminary study started by focusing on the extracellular (EC) portion of human Dsg3. The
$\mathrm{EC}_{1} / \mathrm{EC}_{2}$ domain of Dsg3, spanning from amino acid 1 to 212 , was searched for potential epitopic linear sequences with low redundancy to the self-proteome using the PIR protein database. Matching analysis to the human proteome was performed using pentamer probes, being 5 to 6 amino acids the minimal immunoreactive peptide length [29-31] Redundancy of a peptide sequence is defined here by the number of identical pentamers in common between the analyzed autoantigen and the human proteome.

The Dsg3 sequence was dissected into 5-mer motifs that were used as probes to scan the entire human proteome databank. The Dsg3 pentamers were offset by one residue, i.e. overlapped by four amino acids: MMGLF, MGLFP, GLFPR, LFPRT, FPRTT, etc. The computational analysis produced the histogram reported in Fig. 1. It can be seen that the majority of EC Dsg3 pentamers have a number of perfect match hits in the human proteome, by being shared by a number of other human proteins. Only a few peptide stretches are peculiarly owned by the EC1/EC2 of PV Dsg3 antigen. According to the redundancy hypothesis advanced by our labs [6-13], the non-redundant peptide motifs, i.e. the sequences having a low number of matches (or none at all) in common with the human proteome, might be potentially immunogenic by offering possible epitopic linear determinants unknown to (or rarely seen by) the immune system. In this context, two peptides corresponding to Dsg3 ${ }_{36-44}$ EEMTMQQAK and Dsg3 ${ }_{49-}$ ${ }_{60}$ REWVKFAKPCRE sequences appeared of particular interest, since these fragments present lowest similarity to the human proteins and, additionally, host zero match pentamers. Therefore, the corresponding peptides were synthesized to be used in dot immunoassay with AAbs from PV or PF patient sera. A peptide corresponding to Dsg3 ${ }_{190-204}$ LNSKIAFKIVSQEPA sequence was also synthesized since: i) susceptibility to PV is strongly linked to DRB $1 * 0402$ serotype; ii) the Dsg3 190 -

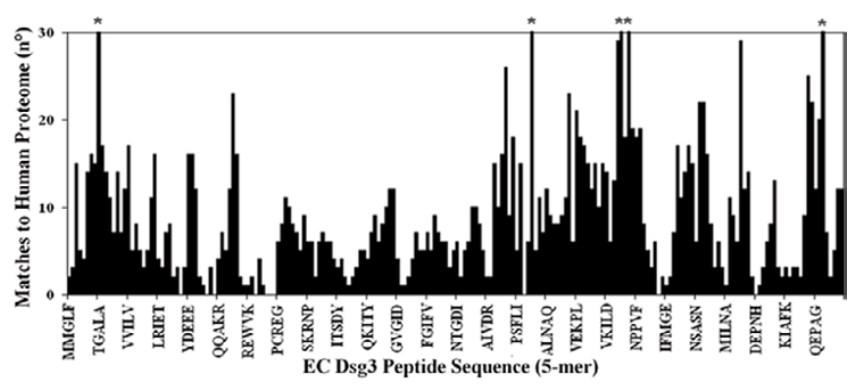

\section{Figure I}

Redundancy profile of the $\mathrm{NH}_{2}$ terminal portion of Dsg3 to human proteome. The ECI/EC2 Dsg3 sequence aal-2I 2 . was scanned for perfect matches to human protein sequences by using pentamers offset by one residue. Asterisk: value $>30$. 
${ }_{204}$ LNSKIAFKIVSQEPA peptide has been reported to bind DRB1*0402; iii) moreover, this peptide appears to be a target for autoreactive T cells in PV patients with active disease [32]. In addition, two Dsg3 synthetic peptides, Dsg3 3 373-380 QVINVREG and Dsg $3_{518-525}$ NRYTGPYT, were used as controls having low redundancy to the human proteome ( 8 and 12 total matches, respectively, in common with the human proteome), but no pentamers with zero similarity to the human proteome. The similarity profile scanning of the Dsg3 synthetic peptides used in dot-immunoassay analyses with sera from PV and PF patients is illustrated in Fig. 2.

\section{Immunoreactivity of non-redundant ECI/EC2 Dsg3 peptides}

The synthetic peptides, selected as above described and illustrated in Fig. 2, were bound to activated nitrocellulose membrane and tested as potential antigenic epitopes against serum from PV patients in dot immunoassays. Because of reports of anti-Dsg immune responses in healthy individuals $[33-35]$, sera from prostate cancer patients were used as controls. Sera from PF patients were used as additional controls.

Fig. 3 shows that the non-redundant $\mathrm{Dsg}_{49-}$ ${ }_{60}$ REWVKFAKPCRE peptide was immunoreactive with all sera from PV. The Dsg3 ${ }_{373-380}$ QVINVREG sequence showed some faint reactivity. The DRB1*0402 binding and T cell epitope Dsg $3_{190-204}$ LNSKIAFKIVSQEPA peptide [32] did not react with any of the human sera used in this study. Interestingly Fig. 3 illustrates that the non-redundant Dsg $3_{49-60}$ REWVKFAKPCRE peptide was immunoreactive with AAbs from PF too.

\section{NMR probing of Dsg3 ${ }_{49-60}$ REWVKFAKPCRE peptide immunoreactivity}

False positives as well as false negatives are a constant feature of immunoassays. Consequently, we controlled the dot-blot results reported in Fig. 3 by NMR spectroscopy to irrefutably demonstrate the immunoreactivity of $\mathrm{Dsg}_{49}$ ${ }_{60}$ REWVKFAKPCRE peptide with both PV and PF AAbs. NMR spectroscopy measures the antigen-antibody binding reaction by monitoring nuclear chemical shifts, i.e. the motional freedom of nuclei. However, application of NMR technology to the analysis of the antigen-antibody interaction is not unproblematic, since the high number of amino acid residues complicates the assignment of specific chemical shift signals. Therefore, we enhanced the NMR detection limit by measuring one-bond protonnitrogen shift correlations with two-dimensional (2-D) phase-sensitive pulsed field gradient HSQC experiments [26]. In addition, synthetic peptides containing ${ }^{15} \mathrm{~N}$ labelled amino acids were used in order to obtain greater intensities and unequivocal resolution. Specifically, the

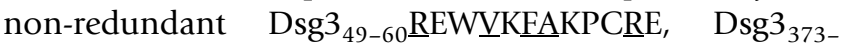

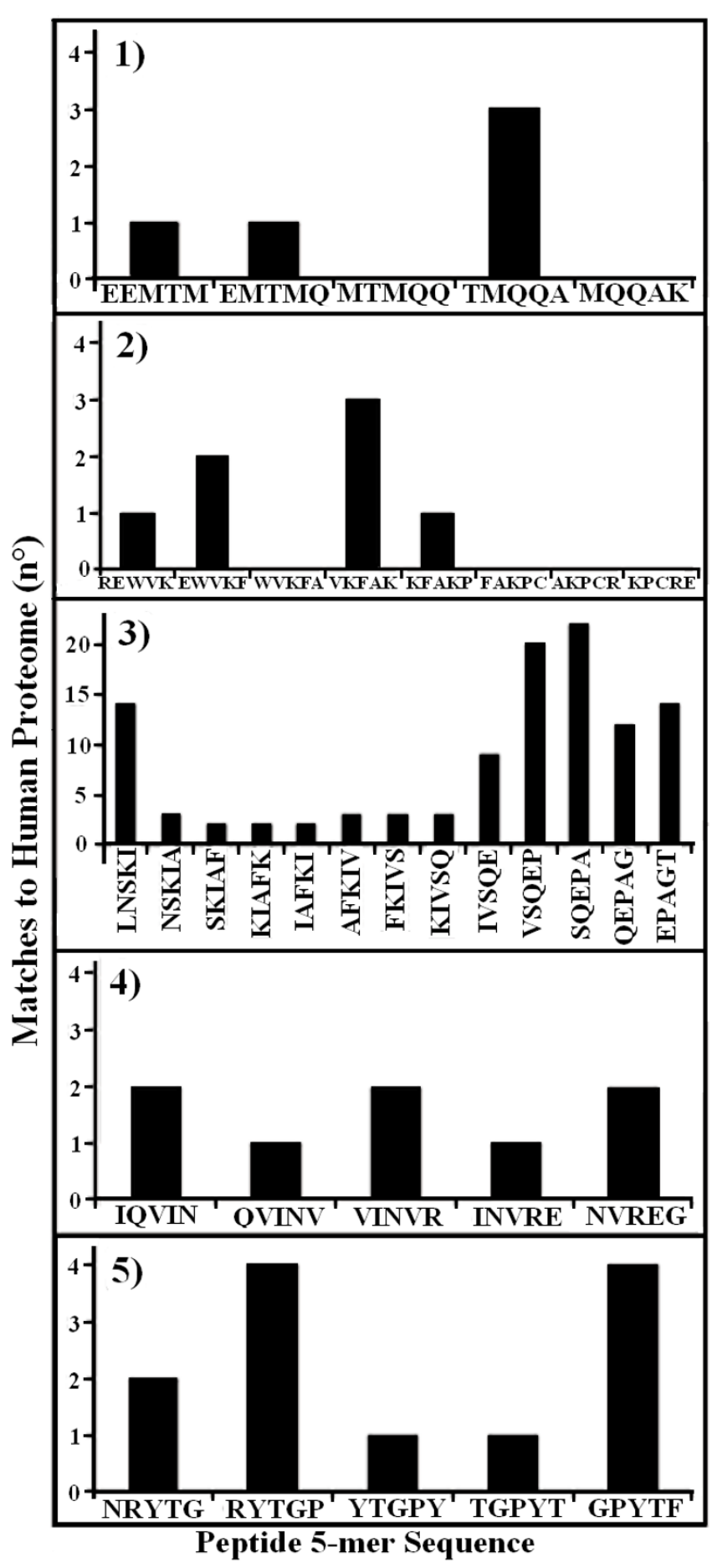

Figure 2

Redundancy scanning on the EC Dsg3 peptide sequences utilized in dot immunoassay analyses with human PV and PF sera. Matching analysis to the human proteome was performed using 5-mer peptide probes. Peptide: I) Dsg3 36 ${ }_{44}$ EEMTMQQAK; 2) Dsg3 ${ }_{49-60}$ REWVKFAKPCRE; 3) Dsg3 190-204 LNSKIAFKIVSQEPA; 4) Dsg3 ${ }_{373-380}$ QVINVREG; 5) Dsg3 $_{518-525}$ NRYTGPYT. 
A) Controls:

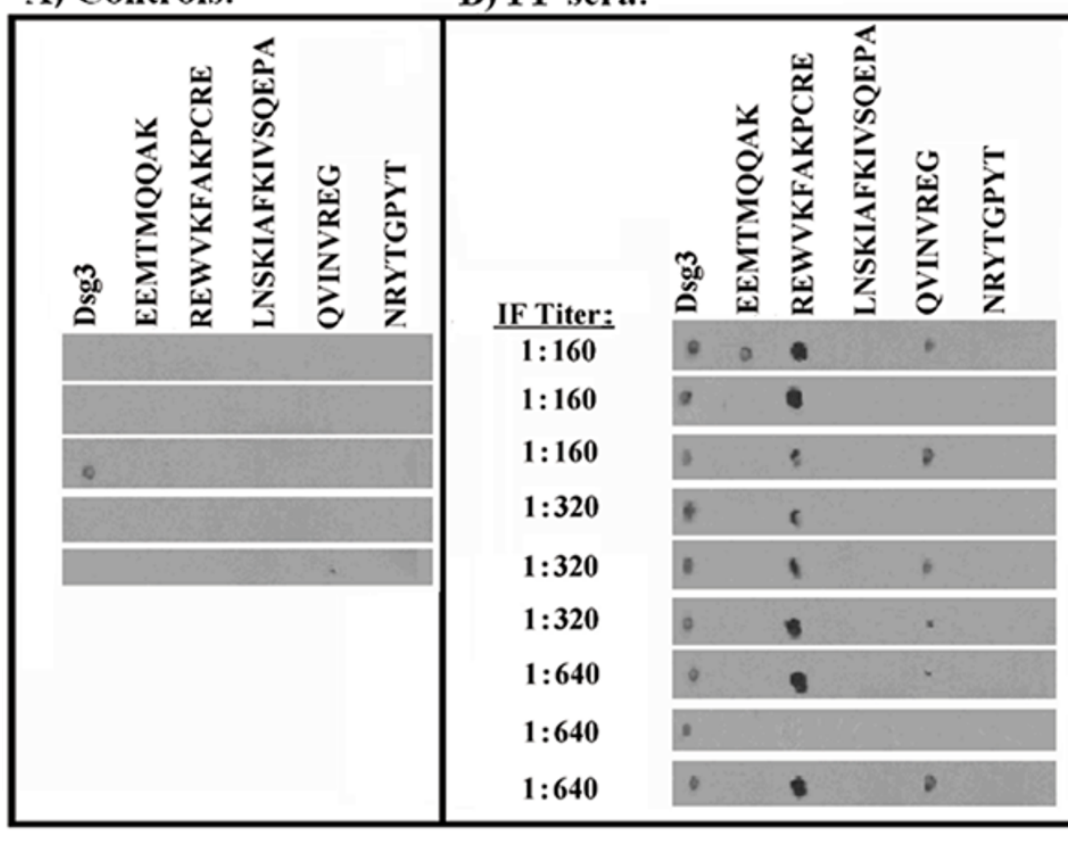

C) PV sera:

\begin{tabular}{|c|c|c|c|c|c|}
\hline IF Titer: & 总 & 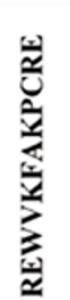 & 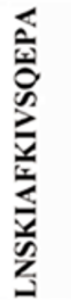 & 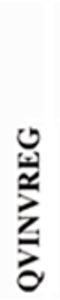 & 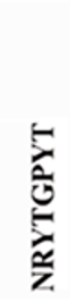 \\
\hline $1: 80$ & 0 & $=$ & & $\cdot$ & \\
\hline $1: 80$ & . & - & & & \\
\hline $1: 320$ & $=$ & $\bullet$ & & & . \\
\hline $1: 320$ & a & - & & - & \\
\hline $1: 320$ & - & - & & & \\
\hline $1: 640$ & - & () & & & \\
\hline $1: 640$ & - & - & & - & \\
\hline $1: 640$ & $\bullet$ & $\bullet$ & & & \\
\hline $1: 640$ & - & - & & $\cdot$ & \\
\hline $1: 10240$ & . & - & & & \\
\hline
\end{tabular}

\section{Figure 3}

Immunoreactivity of PV, PF or prostate cancer human sera towards EC Dsg3 peptides. Sera: A) control sera from prostate cancer patients; B) Pemphigus foliaceous sera; C) Pemphigus vulgaris sera. Immunoreactivity of each serum was assayed by dotblot as described under Methods. Indirect IF titer is reported for each PF and PV serum. Antigens: Dsg3, recombinant EC Dsg 3 protein; peptides with low redundancy to the human proteins: Dsg3 ${ }_{36-44}$ EEMTMQQAK and Dsg3 ${ }_{49-60}$ REWVKFAKPCRE; peptide with high affinity to DRB*0402: Dsg3 ${ }_{190-204}$ LNSKIAFKIVSQEPA; control peptides: Dsg3 ${ }_{373-380}$ QVINVREG and Dsg3 ${ }_{518-}$ ${ }_{52} 5$ NRYTGPYT.

${ }_{380}$ QVINVREG, and Dsg $3_{518-525}$ NRYTGPYT peptides (with ${ }^{15} \mathrm{~N}$-labelled amino acid residues given underlined) were synthesized and chemical shift changes following $\mathrm{AAb}$ addition were monitored in two-dimensional correlated spectroscopy spectra as reported in Fig. 4, 5, and 6, respectively. Each spot in the figures is an NMR signal representing the ${ }^{1} \mathrm{H}-{ }^{15} \mathrm{~N}$ one-bond coupling of the labelled amino acid residues in the peptide.

Table 1 lists the numerical values of the theoretical and experimental chemical shifts of the ${ }^{15} \mathrm{~N}$-labelled amino acid residues in the Dsg $3_{49-60}$ REWVKFAKPCRE peptide. Theoretical values were calculated by using chemical shift statistics from BioMagResBank (www.bmrb.wisc.edu) plus sequence-dependent factors to correct deviations due to local sequence effects [27]. Experimental values derive from the resonance spectrum of the reactions illustrated in Fig. 4, and indicate the chemical shift of the labelled amino acid residues in the free peptides in solution vs. those obtained following AAb addition to the peptide solution.

Specifically, Fig. 4 shows the reaction between human AAbs and the immunoreactive $\mathrm{Dsg}_{49-}$
${ }_{60} \underline{R} E W \underline{V} \underline{F A K P C R E}$ peptide. Fig. 4A reports the Arg, Val, Phe, and Ala selective ${ }^{1} \mathrm{H}-{ }^{15} \mathrm{~N}$ correlations of the free peptide in aqueous solution, with the upper part of Fig. 4A displaying the Arg cross-peak signals due to the $\mathrm{H}$ and $\mathrm{N}$ atoms of amino $\eta$ residues. On the whole, Fig. $4 \mathrm{~A}$ clearly? shows that all of the expected signals are present and in basic agreement with the theoretical data relative to the ${ }^{15} \mathrm{~N}$-labelled amino acids (see chemical shift values in Table 1). The addition of AAbs from control cancer sera did not alter the REW that the spectrum of Fig. 4B could be assigned by reference to the control spectrum of the free peptide reported in Fig. $4 \mathrm{~A}$, both qualitatively and quantitatively. On the contrary, a complete signal deletion was provoked by the addition of AAbs from PF sera as illustrated in Fig.4C, upper and lower parts. Practically, Fig. 4C demonstrates that the PF AAbs were able to specifically neutralize and quantitatively precipitate the low similarity $\mathrm{Dsg}_{49}$ ${ }_{60}$ REWVKFAKPCRE peptide. In the presence of PV AAbs, well resolved resonances for ${ }^{15} \mathrm{~N}$-labelled epitopic REWVKFAKPCRE peptide residues were detectable (Fig. $4 \mathrm{D}$, upper and lower panels), but the signal intensity was much weaker when compared to the control spectra reported in Fig. 4, panels A and B. That indicates that only 


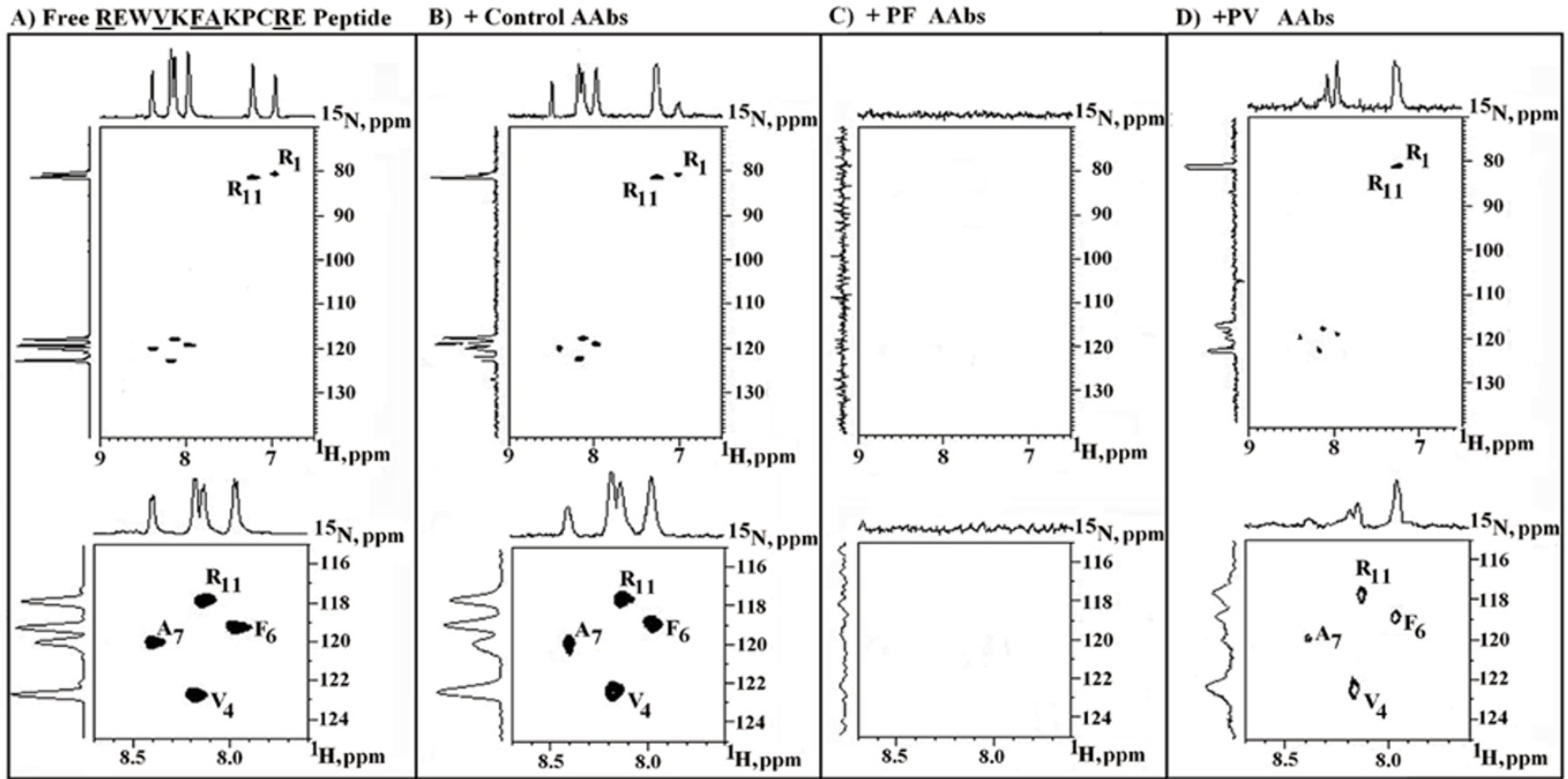

Figure 4

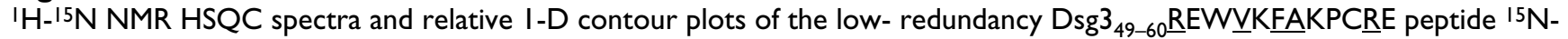
labelled at residues I, 4, 6, 7 and II. A) free peptide in solution; plus AAbs from B) prostate cancer, C) PF, or D) PV patients. Upper panels: portions of the HSQC spectra showing resonances from residues I, 4, 6, 7 and II. Lower panels: expanded region of the HSQC spectra showing resonances from the residues 4, 6, 7 and II, by being I-Arg at terminal position. ${ }^{15} \mathrm{~N}$ labelled residues are given underlined.

a part of the ${ }^{15} \mathrm{~N}$-labelled peptide had been complexed and buried by PV AAbs. No precipitation reaction was observed by using the control ${ }^{15} \mathrm{~N}$-labelled Dsg3 $373-$ ${ }_{380}$ Q $\underline{\text { INVREG }}$ and Dsg $3{ }_{518-525}$ NRYTGPYT peptides (not shown).

\section{Dsg I and Dsg3 cross-react with PF and PV sera: sharing of} the $\mathbf{7 5} \%$ consensus motif REW-KFAK-RE

It is res iudicata in pemphigus pathologies that Dsg3 is the autoantigen of the vulgaris form, whereas Dsg1 is the autoantigen of the foliaceous form [14,15,17-19,33]. In agreement with this statement, in the experiments illustrated in Figs. 3 and 4, PF AAbs were initially used as additional controls. On the other hand, the peculiar data emerging from Figs. 3 and 4 is the highly specific reaction between the PF AAbs preparation and the peptide from PV-associated Dsg3 autoantigen. Indeed, given the caveat that the extent of the ${ }^{15} \mathrm{~N}$-labelled peptide reaction monitored in the NMR spectroscopy analyses depends on the $\mathrm{AAb}$ titer of each of the pooled sera, the NMR spectra reported in Fig. 4 indicated that the reaction extent with AAbs from PF sera was even higher than that monitored with AAbs from PV sera. The finding was of interest also in the light of reports indicating a common desmoglein background in PV and PF, and coexistence of the two pemphigus forms in the same patient [36,37].

To better understand and define the cross-reactivity between the PF AAbs and the PV associated Dsg3 peptide, we analyzed Dsg1 and Dsg3 protein immunoreactivity towards AAbs from PV and PF by Western blot analysis. Fig. 5, panels A and B, show that: i) both PV and PF AAbs recognize a linear desmoglein determinant; ii) the linear desmoglein determinant is common to Dsg1 and Dsg3, so confirming the NMR spectroscopic immunoreaction reported in Fig. 4C.

The cross reaction between the PF AAbs and the PV associated Dsg3 peptide was further investigated by Dsg1/ Dsg3 sequence alignment. The sequence analysis demonstrates $75 \%$ identity in the fragment spanning aa 49 to 60 :

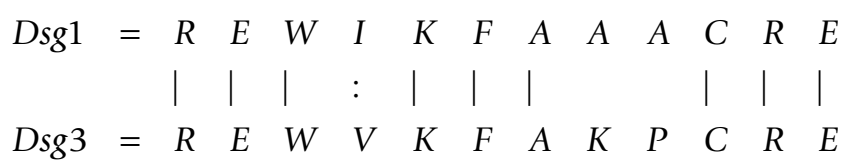

with REW-KFAK-RE as a common consensus motif, and suggests that the shared amino acids of the consensus motif may represent the epitopic residues recognized by 
Table I: Chemical shifts of ${ }^{15} \mathrm{~N}$-labelled residues in $\mathrm{Dsg}_{49-60}$ REWVKFAKPCRE peptide, and changes on control, PV or PF Aab addition

\begin{tabular}{|c|c|c|c|c|c|c|c|c|c|c|}
\hline \multirow[t]{2}{*}{${ }^{15} \mathrm{~N}$-residuel } & \multicolumn{10}{|c|}{ Chemical Shift Values: } \\
\hline & \multicolumn{2}{|c|}{ Theoretical2: } & \multicolumn{4}{|c|}{ Experimental ${ }^{3}$ : } & & & & \\
\hline & \multicolumn{2}{|c|}{ Free peptide } & \multicolumn{2}{|c|}{ Free peptide } & \multicolumn{2}{|c|}{ Control Aabs } & \multicolumn{2}{|c|}{ +PV Aabs } & \multicolumn{2}{|c|}{ +PF Aabs } \\
\hline & ${ }^{\mathrm{I}} \mathrm{H}^{\mathrm{N}}$ & ${ }^{15} \mathrm{~N}$ & ${ }^{\prime} \mathrm{H}^{\mathrm{N}}$ & ${ }^{15} \mathrm{~N}$ & ${ }^{\mathrm{I}} \mathrm{H}^{\mathrm{N}}$ & ${ }^{15} \mathrm{~N}$ & ${ }^{\mathrm{I}} \mathrm{H}^{\mathrm{N}}$ & ${ }^{15} \mathrm{~N}$ & ${ }^{\mathrm{I}} \mathrm{H}^{\mathrm{N}}$ & ${ }^{15} \mathrm{~N}$ \\
\hline I-Arg ${ }^{4}$ & 6.78 & 77.6 & 6.95 & 80.6 & 7.02 & 80.7 & 7.24 & 81.2 & - & - \\
\hline $4-\mathrm{Val}$ & 8.65 & 120.8 & 8.18 & 122.7 & 8.18 & 122.5 & 8.16 & 122.5 & - & - \\
\hline 6-Phe & 8.42 & 121.7 & 7.97 & 119.3 & 7.97 & 119.0 & 7.96 & 119.0 & - & - \\
\hline 7-Ala & 8.29 & 122.3 & 8.40 & 120.0 & 8.40 & 120.0 & 8.38 & 119.8 & - & - \\
\hline \multirow[t]{2}{*}{ II-Arg ${ }^{4}$} & 6.78 & 77.6 & 7.22 & 81.4 & 7.27 & 81.6 & 7.20 & 81.6 & - & - \\
\hline & 8.14 & 119.6 & 8.14 & 117.9 & 8.13 & 117.7 & 8.12 & 117.9 & - & - \\
\hline
\end{tabular}

$115 \mathrm{~N}$-amino acid position in the peptide. ${ }^{2}$ Theoretical chemical shift values were derived from bmrb.wisc.edu, and corrected for deviations due to local sequence effects [27]. ${ }^{3}$ Experimental values from resonance spectra reported in Fig. $4 .{ }^{4}$ Average value of chemical shifts relative to the $\mathrm{H}$ and $\mathrm{N}$ atoms of $\eta$ amino residues in arginine. The chemical shifts relative to the $\mathrm{H}$ and $\mathrm{N}$ atoms of $\alpha$ amino residue are undetectable in I-Arg because of the $\mathrm{NH}_{2}$ terminal position.

both PV and PF sera, so providing a theoretical molecular framework to the data reported in Figs. 3, 4 and 5. Final experimental confirmation was obtained in immunodotblot assays by using the synthetic Dsg $1_{49_{-}}$ ${ }_{60}$ REWIKFAAACRE peptide with PV and PF sera. Fig. 5, panel C, illustrates that PF AAbs recognize both Dsg $1_{49-}$ ${ }_{60}$ REWIKFAAACRE and Dsg3 ${ }_{49-60}$ REWVKFAKPCRE peptides. Likewise, also PV AAbs recognize Dsg $1_{49}$

\section{A) PF AAbs}

\section{B) PV AAbs}

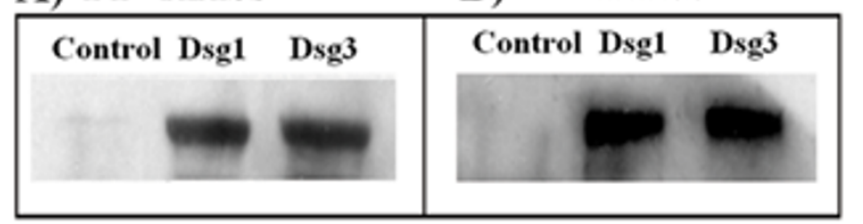

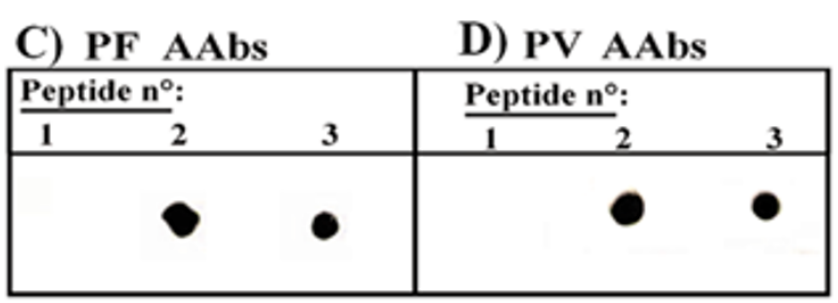

\section{Figure 5}

Cross-reactivity of human recombinant EC DsgI or EC Dsg3 proteins with PF and PV sera. Panels A and B: Western blot analysis of PF or PV serum immunoreactivity towards human recombinant EC Dsgl or EC Dsg3 protein. Control: phosphorylase was used as a control protein. Panels $\mathbf{C}$ and D: Dot blot analysis of PF or PV serum immunoreactivity towards peptide: I) Dsg3 $190-204$ LNSKIAFKIVSQEPA, 2) Dsg3 39-60 REWVKFAKPCRE, and 3) DsgI $_{49-}$ ${ }_{60}$ REWIKFAAACRE.
${ }_{60}$ REWIKFAAACRE as well as Dsg $3_{49-60}$ REWVKFAKPCRE peptide (Fig. 5, panel D).

\section{Discussion}

Using computational biology and proteomics, and applying sequence uniqueness as a search criterium, we have characterized a linear low-redundant Dsg3 segment which is immunorecognized by sera from PV patients as well as PF patients. These data appear of scientific, clinical, and therapeutical interest.

Scientifically, the present study demonstrates that analytical dissection of the human proteome allows to comparatively analyze the molecular basis of complex multifaceted diseases such as the pemphigus, and precisely identify autoantigen portions involved in immune responses. As a matter of fact, the methodology used in this study was extremely effective for individuating one new desmoglein epitope. Sets of 40 synthetic peptides, each 15 residues in length and overlapping by ten amino acid residues, should had been screened to scan the EC1/ EC2 Dsg3 sequence by using canonical peptide-mapping analysis. In our approach identification of an immunodominant linear desmoglein epitope involved only 6 synthetic peptides. The application of the proteomic approach to find unique sequences led to rapid, effective and inexpensive epitope identification when compared to other methods,

Clinically, we note that although other investigators have analyzed desmogleins for patterns of continuous immunoreactive peptides $[19,38,39]$, this is the first time, as far as we know, that a linear determinant on Dsg3 is identified as a precise amino acid sequence immunoreactive with patients' sera. Likewise, this is the first time that a 
common link between PV and PF is presented in terms of a common Dsg3/Dsg1 immunoreactive motif, i.e. the consensus REW-KFAK-RE sequence. Given the caveat that immunogenicity and pathogenicity are not synonymous and further studies are necessary in order to assess the (patho)physiological significance of the present data, undoubtedly these experimental results might be of help in defining the autoantibody response of pemphigus diseases. Indeed, pemphigus is a group of diseases of the skin and mucous membranes that 1) include different forms and variants, 2) are characterized by complex autoantibody profiles, and 3 ) present blistering process as a minimum common clinical denominator [40-42]. Consequently, a major goal in understanding pemphigus autoimmunity is the identification of critical epitopes along the antigen portions involved in the calciumdependent cell-cell adhesion process. In this context, the data presented in this work, describing the NH2 terminal calcium-adhesive Dsg $3_{49-60}$ REWVKFAKPCRE immunoreactive peptide, might represent a prelude to an exact definition of the entire Dsg linear epitope pattern. Indeed, the individuation of the consensus motif as a common sequence recognized by PF and PV sera is an experimental point that might help in the fine definition of the $\mathrm{AAb}$ profile in pemphigo diseases. Moreover, it has to be mentioned that also the monoclonal humoral response to the ectodomain of human Dsg3 targets within the Dsg3 $3_{49-60}$ REWVKFAKPCRE sequence [13]. This is an additional reason for further studying Dsg $3_{49-60}$ sequence in the context of the induction of bullous skin disease resembling PV through passive transfer of PV AAbs [43].

Therapeutically, the usage of proteomic sequence uniqueness as a guiding principle in identifying epitopic sequences along autoantigenic proteins offers the possibility of effective peptide-immunotherapies in autoimmune diseases. The peptide-immunotherapy approach is currently being explored with encouraging results in many autoimmune diseases [44-46]. Indeed, short peptide fragments might be used to selectively block and neutralize autoantibodies, once the effective epitopic sequences have been determined and the definition between immunogenic and pathogenic epitopes is clearly drawn. Moreover, the precise identification of amino acid sequences uniquely present in the autoantigen of interest would also eliminate the side effects deriving from possible crossreactions. In this context, the proteomics-defined REWVKFAKPCRE sequence represents a suitable candidate for a possible peptide-immunotherapy in pemphigus diseases.

\section{Abbreviations}

Dsg, desmoglein; EC, extracellular; PV, Pemphigus vulgaris; PF, Pemphigus foliaceous; HSQC, heteronuclear single quantum correlation; AAbs, autoantibodies.

\section{Authors' contributions}

All Authors have made substantive intellectual contributions to the study conception and design as well as in drafting, modifying and revising the manuscript. In addition, AL has been involved in immunoassays; AAS in sera collection and analysis; DK in NMR studies.

\section{Acknowledgements}

We are grateful to F.P.Fanizzi and M.S. Lin for precious help and critical discussion. Part of the experimental work was carried out at the NMR Unit of Carso Cancer Research Center, Regione Puglia, Italy. This study was supported by Zalmin A.Arlin Cancer Fund, NY, USA (AM); 60\% MIUR, Italy (DK); European Community funding to the PhD course "Carcinogenesis, Aging and Immunoregulation", University of Bari, Italy.

\section{References}

I. Oldstone MBA: Molecular mimicry and immune-mediated diseases. FASEB J 1998, I 2: 1255-1265.

2. Croxford JL, Anger HA, Miller SD: Viral delivery of an epitope from Haemophilus influenzae induces central nervous system autoimmune disease by molecular mimicry. I Immunol 2005, I 74:907-917.

3. Nissim A, Winyard PG, Corrigall V, Fatah R, Perrett D, Panayi G, Chernajovsky $Y$ : Generation of neoantigenic epitopes after posttranslational modification of type II collagen by factors present within the inflamed joint. Arthritis Rheum 2005, 52:3829-3838.

4. Hahn M, Nicholson MJ, Pyrdol J, Wucherpfennig KW: Unconventional topology of self peptide-major histocompatibility complex binding by a human autoimmune $\mathrm{T}$ cell receptor. Nat Immunol 2005, 6:490-496.

5. Carl PL, Temple BR, Cohen PL: Most nuclear systemic autoantigens are extremely disordered proteins: implications for the etiology of systemic autoimmunity. Arthritis Res Ther 2005, 7:RI360-1374.

6. Willers J, Lucchese A, Kanduc D, Ferrone S: Molecular mimicry of phage displayed peptides mimicking GD3 ganglioside. Peptides 1999, 20:1021-1026.

7. Natale C, Giannini T, Lucchese A, Kanduc D: Computer-assisted analysis of molecular mimicry between human papillomavirus 16 E7 oncoprotein and human protein sequences. Immunol Cell Biol 2000, 78:580-585.

8. Dummer R, Mittelman A, Fanizzi FP, Lucchese G, Willers J, Kanduc D: Non-self discrimination as a driving concept in the identification of an immunodominant HMW-MAA epitopic peptide sequence by autoantibodies from melanoma cancer patients. Int J Cancer 2004, I I I:720-726.

9. Kanduc D, Fanizzi FP, Lucchese G, Stevanovic S, Sinha AA, Mittelman A: NMR probing of in silico identification of anti-HPVI 6 E7 mAb linear peptide epitope. Peptides 2004, 25:243-250.

10. Kanduc D: Defining peptide sequences: from antigenicity to immunogenicity through redundancy. Curr Pharmacogenomics 2006, 4:33-37.

II. Lucchese A, Willers J, Mittelman A, Kanduc D, Dummer R: Proteomic scan for tyrosinase peptide antigenic pattern in vitiligo and melanoma: role of sequence similarity and HLA-DR I affinity. J Immunol 2005, I 75:7009-7020.

12. Mittelman A, Tiwari R, Lucchese G, Willers J, Dummer R, Kanduc D: Identification of monoclonal anti-HMW-MAA antibody linear peptide epitope by proteomic database mining. J Invest Dermat 2004, I 23:670-675.

13. Lucchese A, Mittelman A, Lin MS, Kanduc D, Sinha AA: Epitope definition by proteomic similarity analysis: identification of the linear determinant of the anti-Dsg3 MAb 5HIO. J Transl Med 2004, 2:43.

14. Amagai $M$ : Desmoglein as a target in autoimmunity and infection. I Am Acad Dermatol 2003, 48:244-252.

15. Pias EK, Hilario-Vargas J, Li N, Diaz LA: Humoral autoimmunity in pemphigus. Autoimmunity 2004, 37:283-286.

16. Hacker-Foegen MK, Janson M, Amagai M, Fairley JA, Lin MS: Pathogenicity and epitope characteristics of anti-desmoglein-I 
from pemphigus foliaceus patients expressing only IgG autoantibodies. J Invest Dermatol 2003, 12 1:1373-1378.

17. Karpati S, Amagai M, Prussick R, Cehrs K, Stanley JR: Pemphigus vulgaris antigen, a desmoglein type of cadherin, is localized within keratinocyte desmosomes. J Cell Biol 1993, I 22:409-4I5.

18. Kowalczyk AP, Anderson JE, Borgwardt JE, Hashimoto T, Stanley JR, Green KJ: Pemphigus sera recognize conformationally sensitive epitopes in the amino-terminal region of desmoglein-I. J Invest Dermatol 1995, 105: I47-I52.

19. Hashimoto T, Amagai M, Garrod DR, Nishikawa T: Immunofluorescence and immunoblot studies on the reactivity of Pemphigus vulgaris and pemphigus foliaceus sera with desmoglein 3 and desmoglein I. Epithelial Cell Biol 1995, 4:63-69.

20. Nguyen VT, Ndoye A, Shultz LD, Pittelkow MR, Grando SA: Antibodies against keratinocyte antigens other than desmogleins $I$ and 3 can induce Pemphigus vulgaris-like lesions. J Clin Invest 2000, 106: I 467-1479.

21. Grando SA, Pittelkow MR, Shultz LD, Dmochowski M, Nguyen VT: Pemphigus: an unfolding story. J Invest Dermatol 200I, I I 7:990-995.

22. Stanley JR, Nishikawa T, Diaz LA, Amagai M: Pemphigus: is there another half of the story? J Invest Dermatol 200 I, I I 6:489-490.

23. Wu CH, Huang H, Arminski L, Castro-Alvear J, Chen Y, Hu ZZ, Ledley RS, Lewis KC, Mewes HW, Orcutt BC, Suzek BE, Tsugita A, Vinayaka CR, Yeh LS, Zhang J, Barker WC: The Protein Information Resource: an integrated public resource of functional annotation of proteins. Nucleic Acids Res 2002, 30:35-37.

24. Huang $X Q$, Miller $W:$ A time-efficient, linear-space local similarity algorithm. Adv Appl Mathem 1991, 12:337-357.

25. Arteaga LA, Prisayanh PS, Warren SJ, Liu Z, Diaz LA, Lin MS, Cooperative Group on Fogo Selvagem Research: A subset of pemphigus foliaceus patients exhibits pathogenic autoantibodies against both desmoglein-I and desmoglein-3. J Invest Dermatol 2002, I I 8:806-8II.

26. Schleucher J, Schwendinger M, Sattler M, Schmidt P, Schedletzky O, Glaser S], Sorensen OW, Griesinger C: A general enhancement scheme in heteronuclear multidimensional NMR employing pulsed field gradients. J Biomol NMR 1994, 4:30I-306.

27. Schwarzinger S, Kroon GJA, Foss TR, Chung J, Wright PE, Dyson HJ: Sequence-dependent correction of random coil NMR chemical shifts. J Am Chem Soc 200I, I 23:2970-2978.

28. Tsunoda K, Ota T, Aoki M, Yamada T, Yamada T, Nagai T, Nakagawa T, Koyasu S, Nishikawa T, Amagai M: Induction of pemphigus phenotype by a mouse monoclonal antibody against the amino-terminal adhesive interface of desmoglein 3. J Immunol 2003, 170:2170-2178

29. Lindner K, Mole SE, Lane DP, Kenny MK: Epitope mapping of antibodies recognising the $\mathrm{N}$-terminal domain of simian virus large tumour antigen. Intervirology 1998, 41:10-16.

30. Reddehase MJ, Rothbard JB, Koszinowski UH: A pentapeptide as minimal antigenic determinant for MHC class I-restricted $\mathrm{T}$ lymphocytes. Nature 1989, 337:65I-653.

31. Hemmer B, Kondo T, Gran B, Pinilla C, Cortese I, Pascal J, Tzou A, McFarland HF, Houghten R, Martin R: Minimal peptide length requirements for CD4(+) T cell clones-implications for molecular mimicry and T cell survival. Int Immunol 2000, 12:375-383.

32. Wucherpfennig KW, Yu B, Bhol K, Monos DS, Argyris E, Karr RW, Ahmed AR, Strominger JL: Structural basis for major histocompatibility complex (MHC)-linked susceptibility to autoimmunity: charged residues of a single MHC binding pocket confer selective presentation of self-peptides in Pemphigus vulgaris. Proc Natl Acad Sci USA 1995, 92: I 1935-I 1939.

33. Veldman C, Hohne A, Dieckmann D, Schuler G, Hertl M: Type I regulatory $\mathrm{T}$ cells specific for desmoglein 3 are more frequently detected in healthy individuals than in patients with pemphigus vulgaris. I Immunol 2004, I 72:6468-6475.

34. Veldman CM, Gebhard KL, Uter W, Wassmuth R, Grotzinger J, Schultz E, Hertl M: T cell recognition of desmoglein 3 peptides in patients with pemphigus vulgaris and healthy individuals. J Immunol 2004, I 72:3883-3892.

35. Gebhard KL, Veldman CM, Wassmuth R, Schultz E, Schuler G, Hertl M: Ex vivo analysis of desmoglein I-responsive T-helper (Th) $\mathrm{I}$ and Th2 cells in patients with pemphigus foliaceus and healthy individuals. Exp Dermatol 2005, 14:586-592.
36. Harman KE, Gratian MJ, Shirlaw PJ, Bhogal BS, Challacombe SJ, Black $M M$ : The transition of pemphigus vulgaris into pemphigus foliaceus: a reflection of changing desmoglein $I$ and 3 autoantibody levels in pemphigus vulgaris. $\mathrm{Br}$ J Dermatol 2002, | 46:684-687.

37. Feliciani C, Motta A, Castellaneta M, Federica M, De Benedetto A, Toto $P$, Tulli $A$ : Coexisting pemphigus vulgaris and pemphigus foliaceus in the same patient. Int J Dermatol 2005, 44: I39-141.

38. Bhol K, Natarajan K, Nagarwalla N, Mohimen A, Aoki V, Ahmed AR: Correlation of peptide specificity and IgG subclass with pathogenic and nonpathogenic autoantibodies in pemphigus vulgaris: a model for autoimmunity. Proc Natl Acad Sci USA 1995, 92:5239-5243.

39. Hacker MK, Janson M, Fairley JA, Lin MS: Isotypes and antigenic profiles of pemphigus foliaceus and pemphigus vulgaris autoantibodies. Clin Immunol 2002, 105:4-74.

40. Hanakawa Y, Schechter NM, Lin C, Garza L, Li H, Yamaguchi T, Fudaba Y, ishifuji K, Sugai M, Amagai M, Stanley JR: Molecular mechanisms of blister formation in bullous impetigo and staphylococcal scalded skin syndrome. J Clin Invest 2002, I I 0:53-60.

4I. Hanakawa Y, Stanley JR: Mechanisms of blister formation by staphylococcal toxins. J Biochem (Tokyo) 2004, I36:747-750.

42. Payne AS, Hanakawa Y, Amagai M, Stanley JR: Desmosomes and disease: pemphigus and bullous impetigo. Curr Opin Cell Biol 2004, 16:536-543.

43. Anhalt GJ, Labib RS, Voorhees JJ, Beals TF, Diaz LA: Induction of pemphigus in neonatal mice by passive transfer of IgG from patients with the disease. N Engl] Med 1982, 306: I I89-II 96.

44. de Graaf KL, Barth S, Herrmann MM, Storch MK, Otto C, Olsson T, Melms A, Jung G, Wiesmuller KH, Weissert R: MHC class II isotype- and allele-specific attenuation of experimental autoimmune encephalomyelitis. J Immunol 2004, 173:2792-2802.

45. Larche M, Wraith DC: Peptide-based therapeutic vaccines for allergic and autoimmune diseases. Nat Med 2005, I I:S69-76.

46. Goulvestre C, Chereau C, Nicco C, Mouthon L, Weill B, Batteux F: A mimic of p2IWAFI/CIPI ameliorates murine lupus. j Immunol 2005, 175:6959-6967.
Publish with Bio Med Central and every scientist can read your work free of charge

"BioMed Central will be the most significant development for disseminating the results of biomedical research in our lifetime. "

Sir Paul Nurse, Cancer Research UK

Your research papers will be:

- available free of charge to the entire biomedical community

- peer reviewed and published immediately upon acceptance

- cited in PubMed and archived on PubMed Central

- yours - you keep the copyright
BioMedcentral 